\title{
1 First-principles calculations of the lattice thermal conductivity of the lower mantle
}

2 Stephen Stackhouse ${ }^{a, k}$, Lars Stixrude ${ }^{b}$, Bijaya, B. Karki ${ }^{c}$

3 a School of Earth and Environment, University of Leeds, Leeds LS2 9JT, United Kingdom.

${ }^{\mathrm{b}}$ Department of Earth Sciences, University College London, Gower Street, London WC1E 6BT, United Kingdom.

${ }^{c}$ School of Electrical Engineering and Computer Science, Department of Geology and Geophysics, and Center for Computation and Technology, Louisiana State University, Baton Rouge, LA70803, United States of America.

*E-mail addresses: s.stackhouse@leeds.ac.uk; l.stixrude@ucl.ac.uk; karki@csc.Isu.edu

\section{Abstract}

13 The temperature variations on top of the core-mantle boundary are governed by the thermal conductivity of the minerals that comprise the overlying mantle. Estimates of the thermal conductivity of the most abundant phase, $\mathrm{MgSiO}_{3}$ perovskite, at core-mantle boundary conditions vary by a factor of ten. We performed ab initio simulations to determine the lattice thermal conductivity of $\mathrm{MgSiO}_{3}$ perovskite, finding a value of $6.8 \pm 0.9$ $\mathrm{W} \mathrm{m}^{-1} \mathrm{~K}^{-1}$ at core-mantle boundary conditions (136 GPa and $4000 \mathrm{~K}$ ), consistent with geophysical constraints for the thermal state at the base of the mantle. Thermal conductivity depends strongly on pressure, explaining the dynamical stability of superplumes. The dependence on temperature and composition is weak in the deep mantle: our results exhibit saturation as the phonon mean free path approaches the interatomic spacing. Combining our results with seismic tomography, we find large lateral variations in the heat-flux from the core that have important implications for core dynamics.

\section{Keywords}

$\mathrm{MgSiO}_{3}$ perovskite; thermal conductivity; mantle dynamics 


\section{Introduction}

Heat-flux at the core-mantle boundary has important implications for the thermal evolution of the core and mantle (Lay et al., 2008), the size and stability of plumes (Dubuffet et al., 1999), and generation of the magnetic field (Gubbins et al., 2011). Despite this, there is a wide range of estimates of the thermal conductivity of the lower mantle (Osako and Ito, 1991; Hofmeister, 2008; Goncharov et al., 2010; de Koker, 2010; Manthilake et al., 2011; Haigis et al., 2012; Ohta et al., 2012; Dekura et al., 2013; Tang et al., 2014; Ammann et al., 2014; Ohta et al., 2014). As insulators and semi-conductors, the major lower mantle phases: (Mg, $\mathrm{Fe}) \mathrm{SiO}_{3}$ perovskite, $\mathrm{CaSiO}_{3}$ perovskite, and $(\mathrm{Mg}, \mathrm{Fe}) \mathrm{O}$ ferropericlase are expected to conduct heat via phonons (lattice vibrations), but experimental and theoretical studies of their lattice thermal conductivity have suffered significant limitations.

Technical constraints mean that experimental measurements are limited to temperature much lower than those in the deep Earth, and thus long extrapolations must be made to estimate values in the lowermost mantle. In addition, while the results of ambient temperature studies of $\mathrm{MgSiO}_{3}$ perovskite (Osako and Ito, 1991; Ohta et al., 2012) are in reasonable agreement, measurements at elevated temperature (500-1100 K) and $26 \mathrm{GPa}$ (Manthilake et al., 2011), suggests a $300 \mathrm{~K}$ lattice thermal conductivity almost twice as large. This suggests that there are discrepancies even at low temperature and pressure.

Classical simulations, where simple functional forms define interactions between atoms, also have considerable uncertainties. Haigis et al. (2012) used a classical potential to compute lattice thermal conductivity via equilibrium molecular dynamics and Green-Kubo relations, while Ammann et al. (2014) used a classical potential to calculate lattice thermal conductivity via non-equilibrium molecular dynamics simulations. The difference of up to a factor of two between the studies, for the lattice thermal conductivity of $\mathrm{MgSiO}_{3}$ perovskite, illustrates the uncertainty due to the choice of classical potential. This source of 
uncertainty has been highlighted in other studies (Chen et al., 2012; Howell, 2012) and shows the importance of performing ab initio calculations, where forces are calculated from first-principles.

Previous ab initio calculations of the lattice thermal conductivity of $\mathrm{MgSiO}_{3}$ perovskite have also suffered important limitations. Dekura et al. (2013) and Tang et al. (2014) performed lattice dynamics calculations, which are limited by the assumption that anharmonic terms are truncated at third-order. The truncation is significant, because it requires the lattice thermal conductivity to vary as the inverse of the temperature $\left(T^{1}\right)$, more rapidly than observed in silicates and oxide perovskites at temperatures greater than the Debye temperature (Marquardt et al., 2009a; Hofmeister, 2010), and leads to underestimation of the conductivity at high temperature.

Here, we take a different approach, calculating the lattice thermal conductivity of $\mathrm{MgSiO}_{3}$ perovskite using the 'direct' non-equilibrium molecular dynamics method (NEMD), with forces calculated directly from density functional theory. The direct method, which we have used previously to compute the lattice thermal conductivity of $\mathrm{MgO}$ periclase (Stackhouse et al., 2010), has the advantage that anharmonicity is fully included with no truncation. The method is conceptually straightforward: lattice thermal conductivity is calculated from Fourier's law by computing the temperature gradient induced by an imposed heat-flux.

\section{Theory}

\subsection{Non-Equilibrium Molecular Dynamics Simulations}

The lattice thermal conductivity of $\mathrm{MgSiO}_{3}$ perovskite was calculated using ab initio nonequilibrium molecular dynamics (Stackhouse and Stixrude, 2010). The method is intuitive, following the design of experimental techniques. The simulation cell is divided up into sections (Fig. 1(a)). One section is designated the 'hot section' and another the 'cold section'. These are separated by a distance of half the length of the simulation cell. At 
regular intervals heat is transferred from the cold section to the hot section, generating a heat-flux. Over time, a temperature gradient develops between the hot and cold sections (Fig. 1(b)). Once steady state is reached thermal conductivity is calculated from Fourier's law:

$$
k=\frac{\langle J(t)\rangle}{\langle d T / d x\rangle}
$$

where $k$ is the thermal conductivity, and $\langle J(t)\rangle$ and $\langle d T / d x\rangle$ are the time average of the heatflux and the temperature gradient.

In order to conserve the total kinetic energy and linear momentum of the system, the transfer of energy from the cold section to the hot section is achieved by assigning the hottest atom in the cold section and coldest atom in the hot section the velocities that would arise from a hypothetical elastic collision between them (Müller-Plathe, 1997; NietoDraghi and Avalos, 2003).

The temperature gradient is determined from a linear fit to the temperature of the individual sections. Due to the periodic nature of the simulation, heat enters and leaves from both sides of the hot and cold sections and two temperature gradients develop, leading to a temperature profile resembling that shown in Fig. 1(b). Rather than discard one of them, the temperature of symmetrically equivalent sections is averaged. Due to the non-Newtonian nature of the energy transfer, the temperature gradient is non-linear around the hot and cold sections. In view of this, these sections are excluded from the fit to determine the temperature gradient (Supplementary Material Fig. S1(d)).

\subsection{Finite-Size Effects}

Our analysis of finite-size effects follows that of Schelling et al. (2002) and has been used widely as a means of obtaining results in the limit of infinite systems (Zhou et al., 2009; 
101 Sellan et al., 2010; Howell, 2011a, 2011b, 2012; Hu et al., 2011). Kinetic theory relates

102 thermal conductivity to phonon mean free path

103

$$
k=\frac{1}{3} C_{v} v l
$$

where $C_{v}$ is the volumetric heat capacity, $v$ is the mean sound velocity and $l$ is the phonon mean free path. Since phonons are scattered within the hot and cold sections, the longest possible phonon mean free path is equal to the distance between them, i.e. half the length of the simulation cell. If the true phonon mean free path is much longer than this, the thermal conductivity calculated will be significantly underestimated. scattering mechanisms: phonon-phonon scattering and phonon-boundary scattering

111 occurring at the hot and cold sections, then the effective mean free path for a simulation

112 cell of length $L$ is

$$
l_{L}^{1}=l_{p h}^{1}+l_{b}^{1}
$$

114 where $l_{p h}$ is the contribution from phonon-phonon scattering and $l_{b}$ the contribution from

115 phonon-boundary scattering. $l_{b}$ is assumed to be $L / 4$, where $L$ is the total length of the

116 simulation cell. The factor of 4 arises because phonons originating between the hot and

117 cold sections will travel, on average, a quarter of the simulation cell length before

118 encountering the hot and cold sections. Substituting Eq. (3) into (2) and rearranging leads

119 to

$$
k_{L}{ }^{1}=k^{1}+b L^{1}
$$

121 where $k_{L}$ is the thermal conductivity calculated for a simulation cell of length $L, k_{\infty}$ the

122 thermal conductivity of a simulation cell of infinite length (i.e. the true value) and $b$ a 123 constant equal to 


$$
b=\frac{12}{C_{v} v}
$$

125 Thus by calculating the thermal conductivity for simulation cells of different length and

126 plotting against $L^{-1}$, one can estimate $k^{1}$.

127 Previous studies (Sellan et al., 2010; Howell, 2012) have shown that the linear

128 extrapolation (Eq. (4)) may be inaccurate when the phonon mean free path is much longer

129 than the smallest simulation cell. For example, studies of silicon near room temperature,

130 which has a long phonon mean free path, require simulation cells on the order of 10000

131 atoms. In the case of our calculations, smaller simulations cells are sufficient, since the

132 temperature is much higher and the phonon mean free path much shorter (Tadano et al.,

133 2014). In our previous calculations of periclase (Stackhouse et al., 2010), we used

134 simulation cells of a similar size, and found our results to be in good agreement with other

135 theoretical methods and experimental results, and the phonon mean free path to be

136 shorter than the smallest simulation cell.

137 Phonon mean free paths can be estimated from the slope of the linear relationship

138 between $k_{L}{ }^{1}$ and $L^{-1}$, combining Eqs. (2) and (5)

$$
l=\frac{b k}{4}
$$

140 Estimated values of $l$ (Supplementary Material Table S1) are shorter than the shortest

141 simulation cell used at all temperature and pressures studied, indicating that our

142 simulation cells are large enough to obtain accurate results.

143 Calculations may also be inaccurate if the cross-sectional area of the simulation cell is

144 too small (Schelling et al., 2002; Zhou et al., 2009; Hu et al., 2011). This is because the

145 phonon population is then biased towards those propagating in the long direction of the

146 simulation cell, leading to an overestimate of the thermal conductivity. This is not expected 
147 to be a serious issue in our simulations, because the phonon mean free path is

148 comparable to the cross-sectional dimensions. To test this issue, we have performed

149 simulations with different cross-sectional areas at $110 \mathrm{GPa}$ and $1000 \mathrm{~K}$ and $110 \mathrm{GPa}$ and

$1503250 \mathrm{~K}$. The results are identical within uncertainty (Supplementary Material Table S1).

\section{Calculation Details}

152 Calculations were performed using a version of the density functional theory based VASP 153 code (Kresse and Furthmuller, 1996a, 1996b) modified to perform NEMD. The local

154 density approximation (Perdew and Zunger, 1981) was used for the exchange-correlation

155 functional. Ultrasoft pseudopotentials were employed, with valence electron

156 configurations: $3 s^{2}$ for $\mathrm{Mg}, 3 s^{2} 3 p^{2}$ for Si and $2 s^{2} 2 p^{4}$ for O. The kinetic-energy cut-off for the

157 plane-wave basis set was set to $400 \mathrm{eV}$ and Brillouin zone sampling was restricted to the

$158 \Gamma$-point. Fermi-smearing was applied in all calculations, with a broadening-width equal to

159 the simulation temperature. The convergence criteria for the self-consistency loop was $10^{-4}$

$160 \mathrm{eV}$. The time-step was set to $1 \mathrm{fs}$ and the Nosé thermostat was employed to maintain a

161 constant temperature (Nosé, 1984). By calculating the forces from first-principles, we

162 avoid issues associated with empirical pair potentials (Chen et al., 2012; Howell, 2012).

163 The dimensions of all simulation cells used in the current work are listed in

164 Supplementary Material Table S2. The cell parameters at each pressure and temperature $165(P-T)$ point, were determined from equilibrium molecular dynamics simulations using 80 166 atom $\mathrm{MgSiO}_{3}$ models.

167 Before each NEMD calculation, the simulation cell was equilibrated by performing 1 ps 168 of equilibrium molecular dynamics, after which energy exchange was initiated. The energy 169 exchange periods used are listed in Supplementary Materials Table S2. These were 170 chosen to produce a temperature difference of $500-1000 \mathrm{~K}$ between the hot and cold 171 sections and took values of between $40-80$ fs. Simulations using longer exchange periods 
172

173

converged more slowly, and led to values with larger associated uncertainties, because the error in the temperature gradient increased. Initial tests showed that, within a certain range, using different exchange periods had little effect on the results (Supplementary Materials Fig. S2).

Most NEMD calculations were run for a minimum of about 50 ps (Supplementary Material Table S2), at least 10 ps of which was allowed for steady state to be reached. Thermal conductivity was calculated using the remaining portion. In general, this led to converged values for the heat-flux, temperature gradient and thermal conductivity (Supplementary Material Fig. S1). When a simulation was judged not to have converged fully i.e. the time average of the thermal conductivity had not have flattened out, simulations were run longer.

The uncertainty in the time average of the heat-flux was determined using the appropriate statistics (Flyvbjerg and Petersen, 1989), taking into account correlation. The same method was also used to compute the uncertainty in the time average of the temperature of individual sections. To calculate the temperature gradient the temperatures of symmetrically equivalent sections were averaged and fit using weighted least square regression, but excluding the values for the hot and cold sections and those either side of them (Fig. S1(d)). The thermal conductivity for a simulation cell of infinite length was determined from a weighted least squares fit to a plot of inverse thermal conductivity against inverse simulation cell length (Supplementary Material Figs. S3 and S4). The extrapolated values are listed in Supplementary Material Table S1.

\section{Results}

At lower mantle conditions, we find that the temperature dependence of the thermal conductivity is weak: at $75 \mathrm{GPa}$, lattice thermal conductivity decreases from $5.3 \pm 0.7 \mathrm{Wm}$ ${ }^{1} \mathrm{~K}^{-1}$ at $2500 \mathrm{~K}$ to $4.7 \pm 0.8$ at $4000 \mathrm{~K}$ (Fig. 2). Note that, our results indicate that the lattice 
197 thermal conductivity of $\mathrm{MgSiO}_{3}$ perovskite is isotropic at $75 \mathrm{GPa}$ and $2500 \mathrm{~K}$ to within the

198 uncertainty of our results (Fig. S3 and Table S1), and this is presumed to be the case at all

199 other conditions. All values shown in Figs. 2 and 3 are for the [100] direction. Both here and throughout the manuscript the uncertainties indicate the standard error. This variation is weaker than the often-assumed $T^{-1}$ dependence, and even weaker than the $T^{2 / 5}$ dependence recently suggested for $\mathrm{MgSiO}_{3}$ perovskite, based on experimental results at lower pressures and temperatures (Manthilake et al., 2011). Such weak temperature dependence indicates saturation (Roufosse and Klemens, 1974; Marquardt et al., 2009a; Hofmeister, 2010), where the phonon mean free path approaches the inter-atomic spacing. This means that pressure (or density) dependence dominates throughout most of the lower mantle: at $4000 \mathrm{~K}$, lattice thermal conductivity increases from $5.3 \pm 0.7 \mathrm{Wm}^{-1} \mathrm{~K}^{-1}$ at $75 \mathrm{GPa}$ to $9 \pm 2 \mathrm{Wm}^{-1} \mathrm{~K}^{-1}$ at $145 \mathrm{GPa}$ (Fig. 2).

Our results agree well with room temperature experimental data (Osako and Ito, 1991;

210 Ohta et al., 2012; Ohta et al. 2014), showing comparable pressure dependence. The 211 measurements of Manthilake et al. (2011), show similar temperature dependence, but are 212 higher by about $5 \mathrm{Wm}^{-1} \mathrm{~K}^{-1}$ (Fig. 2 (main)). We note that their results also disagree with the 213 ambient temperature values of Ohta et al. (2012). Lattice dynamics calculations of Dekura 214 et al. (2013) agree with our results at low temperature, as expected, but predict a value 215 half that of our study at core-mantle boundary conditions. We attribute this difference to 216 the truncation of anharmonic terms at third order in the lattice dynamics calculation, which 217 assumes $T^{-1}$ behavior and does not capture the saturation (Roufosse and Klemens, 1974) 218 that we find at high temperature. The values of Tang et al. (2014), which also suffer from 219 truncation of anharmonic terms, are much lower than all other studies. It has been 220 proposed that finite-size effects in NEMD simulations lead to erroneous values (Haigis et 221 al., 2012), but this issue is expected to be minimal for $\mathrm{MgSiO}_{3}$ perovskite at lower mantle conditions, where the phonon mean free path is short and lattice thermal conductivity is 
223 low (Supplementary Material Fig. S4 and Table S1) (Tadano et al., 2014). Indeed, 224 previous simulations based on classical potentials and much larger systems show 225 remarkably similar values of the thermal conductivity at the core-mantle boundary 226 (Ammann et al., 2014).

\section{Discussion}

228 Our results serve as a test of approximate theories of the density and temperature 229 dependence of the thermal conductivity. Our results disagree with the commonly assumed $230 T^{1}$ or $T^{1 / 2}$ temperature dependence. Instead, we find that our results are reproduced well 231 by a model (Supplementary Material Section S1) that combines the Leibfried-Schlömann 232 equation with temperature dependence that includes the effects of saturation (Roufosse 233 and Klemens, 1974)

$$
k \propto \frac{M^{1 / 3} 3}{{ }^{2} T}\left\{\frac{2}{3}\left[\frac{T_{S}()}{T}\right]^{1 / 2}+\frac{1}{3}\left[\frac{T}{T_{S}()}\right]\right\} C
$$

where $M$ is the mean atomic mass, $\Omega$ mean atomic volume, $\theta$ Debye temperature, $\gamma$

Grüneisen parameter, $T$ temperature, and $T_{S} \mu M \Omega^{2 / 3} \theta^{2} / \gamma^{2}$ is the temperature at which saturation becomes significant. The term in the brackets accounts for saturation and causes $k$ to vary more weakly than $T^{1 / 2}$ at high temperature, in excellent agreement with our results. The heat capacity $C$, accounts for phonon population effects at low temperature. All quantities are computed from a thermodynamic model (Stixrude and 241 Lithgow-Bertelloni, 2011). The two constants of proportionality are chosen by fitting to our NEMD results.

243 We estimate the lattice thermal conductivity across the lower mantle, arriving at a value 244 of $8.1 \pm 1.1 \mathrm{Wm}^{-1} \mathrm{~K}^{-1}$ at the core-mantle boundary (Fig. 3), by combining the results of the 245 present work, with our earlier ab initio predictions for periclase (Stackhouse et al., 2010), 
and scaling laws for minor phases and impurities (Supplementary Material Sections S2 to S4). We approximate the lower mantle as pyrolite: (Mg,Fe) $\mathrm{SiO}_{3}$ perovskite (75 percent), $\mathrm{CaSiO}_{3}$ perovskite (6 percent) and (Mg,Fe)O ferropericlase (19 percent) (Stixrude and Lithgow-Bertelloni, 2011). Recent experimental results show that iron impurities greatly reduce lattice thermal conductivity (Manthilake et al., 2011), at least at the low temperatures at which the measurements were made. By assuming this same large reduction, previous studies (Manthilake et al., 2011; Haigis et al., 2012) arrived at values for the lattice thermal conductivity of the lower mantle similar to ours. However, we expect the impact of impurities to be reduced at lower mantle temperatures. Based on the theory of Klemens (1960), we estimate the fractional lowering of the lattice thermal conductivity of the lower mantle due to iron impurities to be 8 percent at the core-mantle boundary, i.e. our value at the core-mantle boundary in the iron-free limit is very similar: $8.8 \pm 1.2 \mathrm{Wm}^{-1} \mathrm{~K}^{-}$

1. The influence of aluminum is expected to be less than that of iron (Ohta et al., 2014).

Our value for an iron-free lower mantle is substantially smaller than that estimated in a recent experimental study (Manthilake et al., 2011) which we attribute to the long extrapolation from the experimental results to lower mantle conditions. Our value is a factor of 2 larger than the estimate based on the lattice dynamics calculations of Dekura et al. (2013) and a factor of 6 larger than that based on the lattice dynamics calculations of 264 Tang et al. (2014), which we attribute to the truncation of anharmonic terms in these 265 studies.

266 The thermal conductivity of iron-bearing phases may be influenced by electronic 267 transitions at lower mantle conditions. The influence of the high-spin to low-spin transition 268 on thermal conductivity is unknown. If we assume that the primary effect of the transition is 269 to decrease the atomic spacing, and take the volume decrease of Tsuchiya et al. (2006), based on scaling relations (Supplementary Material Section S2), the lattice thermal 
272 high-spin state. Within the transition region, in which high-spin and low-spin iron coexists 273 in variable amounts, the mean acoustic wave velocity is reduced. If we assume that bulk 274 sound velocity is reduced by the amount reported by Wentzcovitch et al. (2009) and that 275 the shear velocities are unaffected (Marquardt et al., 2009b), based on scaling relations 276 (Supplementary Material Section S2), the lattice thermal conductivity may be 15 percent smaller than the high-spin phase. The influence of the spin transition of the lattice thermal conductivity of perovskite will be much less than in ferropericlase, because of the lower iron content and the smaller fraction of ferrous iron. The spin transition appears to decrease photon thermal conductivity and reduce the electrical conductivity (Goncharov et al., 2010). Although pure FeO becomes metallic at high pressures and temperatures (Fischer et al., 2011), thermal transport by electrons is unlikely to contribute significantly to thermal conductivity for plausible mantle iron concentrations. Extreme enrichment in iron, i.e. Fe/Si 1, may produce much greater thermal conductivity by stabilizing new phases in which heat transport by electrons becomes important (Manga and Jeanloz, 1996), but seismic evidence rules out such extreme enrichments, even in ultra-low velocity zones (Rost et al., 2005).

In addition to scattering from impurities, scattering from interactions with electrons and grain boundaries can also influence lattice thermal conductivity. In the lower mantle, $(\mathrm{Mg}, \mathrm{Fe}) \mathrm{SiO}_{3}$ perovskite, $\mathrm{CaSiO}_{3}$ perovskite and $(\mathrm{Mg}, \mathrm{Fe}) \mathrm{O}$ ferropericlase are insulators and semiconductors. Older studies of semiconductors (e.g. Boghosian and Dubey, 1978) suggest that phonon-electron scattering is only important at low temperature $(<5 \mathrm{~K})$, but a more recent first-principles investigation (Liao et al., 2015) shows that, for silicon with high carrier concentrations $\left(>10^{19} \mathrm{~cm}^{-3}\right)$, it has a marked effect at room temperature. There are no studies of the effect of phonon-electron scattering at lower mantle conditions, but we note that the analysis of Liao et al. (2015) indicates that phonon-electron scattering is most significant for phonons with a mean free path greater than $100 \mathrm{~nm}$. This is much longer 
than that expected for phonons in $\mathrm{MgSiO}_{3}$ perovskite (Supplementary Material Table S1) and $\mathrm{MgO}$ periclase (Stackhouse et al. 2010), at lower mantle conditions. Inclusion of impurities will reduce the phonon mean free paths of the phases further. In view of this, we conclude that the effect of phonon-electron scattering is negligible in the lower mantle.

The effect of phonon-boundary scattering on lattice thermal conductivity becomes important when the mean free path is comparable to the grain size. Imada et al. (2014) showed that, at $300 \mathrm{~K}$, there is a significant difference between single- and poly-crystal lattice thermal conductivity measurements for $\mathrm{MgO}$ periclase, in particular, at high pressure where the phonon mean free path is long in the single-crystal. Their analysis suggests that at core-mantle boundary conditions the lattice thermal conductivity of the phase will be independent of grain size, because of the much shorter mean free path. Since the mean free path of phonons in $\mathrm{MgSiO}_{3}$ perovskite and iron-bearing phases is expected to be even shorter than that of $\mathrm{MgO}$ periclase, the lattice thermal conductivity of the lower mantle should be independent of grain size.

In some regions of the lower few hundred kilometers of the mantle, it is expected that perovskite transforms to post-perovskite (Wookey et al., 2005). Some studies indicate that the lattice thermal conductivity of post-perovskite is a factor of two greater than that of perovskite (Ohta et al., 2012; Ammann et al., 2014), but others suggest that this is only true at the low temperatures at which the experiments were conducted and at high temperature the difference is much smaller (Haigis et al., 2012). In view of this, we ignore differences in the lattice thermal conductivities of the two phases. We also neglect possible radiative contributions to the thermal conductivity, because measurements show that these are less than $0.5 \mathrm{Wm}^{-1} \mathrm{~K}^{-1}$ (Goncharov et al., 2008). However, we note that a consensus has not yet been reached: another experimental study concludes that the radiative contribution could be up to $5 \mathrm{Wm}^{-1} \mathrm{~K}^{-1}$ (Keppler et al., 2008). 
323 Our predicted value of the thermal conductivity at the core-mantle boundary is consistent 324 with a variety of geophysical constraints (Lay et al., 2008). A boundary layer analysis 325 (Supplementary Material Section S4) shows that for a thermal boundary layer thickness $\delta$ $326 \sim 100 \mathrm{~km}$ (Fig. 4), our results satisfy independent constraints on the temperature across 327 the thermal boundary layer from extrapolation of the mantle geotherm and inner-core 328 freezing; the location of the seismic discontinuities caused by the perovskite to post329 perovskite phase transition; and bounds on the heat-flow from intra-plate volcanism and 330 heat conducted down the core adiabat.

331 The increase in thermal conductivity with increasing depth (Fig. 3) that we find - nearly a 332 factor of two across the lower mantle - is dynamically significant. Greater thermal 333 conductivity at depth is essential for stabilizing so-called superplumes: large-scale 334 structures in the bottom-most $1000 \mathrm{~km}$ of the mantle beneath Africa and the Pacific, with 335 very low shear wave velocity indicating higher than average temperature (Dziewonski et 336 al., 2010). Dynamical simulations (Dubuffet et al., 1999) show that the buoyant instability 337 of hot superplumes can be counter-acted and their long-term stability explained if the 338 thermal conductivity of the lower mantle is higher than the rest of the mantle, just as we 339 find.

340 While the physical model outlined in the previous sections completely specifies the 341 calculation of the lattice thermal conductivity at all pressure and temperature conditions, 342 we recognize that it may be useful to have a simpler, approximate form that captures the 343 essence of these results. We present a simple approximation to the variation of the lattice 344 thermal conductivity of pyrolite with pressure and temperature that is value across the 345 lower mantle regime

$$
k=(4.9 \mathrm{GPa}+0.105 P) f \frac{T}{1200 K} \div \frac{1200 K}{T} \div
$$


where $k$ is thermal conductivity $\left(\mathrm{Wm}^{-1} \mathrm{~K}^{-1}\right), P$ is pressure (GPa), $T$ is temperature $(\mathrm{K})$ and $f$ 348 is a function based on the theory of Roufosse and Klemens (1974), which accounts for 349 saturation (Supplementary Material Section S1). This equation reproduces our results for pyrolite to within 10 percent along mantle geotherms with potential temperatures between

$3511000 \mathrm{~K}$ and $2000 \mathrm{~K}$ and with or without a bottom thermal boundary layer and over the 352 entire lower mantle pressure regime.

353 According to our results the thermal conductivity in the lower mantle is nearly 354 homogeneous laterally, because of the weak dependence that we find on temperature and 355 impurities. For example, varying the temperature by $\pm 500 \mathrm{~K}$, changes the thermal 356 conductivity at the core-mantle boundary by only 5 percent. Superplumes may also be 357 stabilized by chemical heterogeneity; indeed seismic evidence of sharp sides indicates 358 that they have a different chemical composition from normal mantle (Ni et al., 2002). We 359 find that, in the lower mantle, the influence of chemical heterogeneity on thermal 360 conductivity is minor (e.g. doubling the Fe/Mg ratio decreases the conductivity by only 5 361 percent). This idea was also suggested by Manthilake et al. (2011), who showed that iron 362 concentration has little effect on the lattice thermal conductivity of $(\mathrm{Mg}, \mathrm{Fe}) \mathrm{O}$ ferropericlase, 363 although we note that they estimate a much larger difference between an iron-free and 364 iron-bearing lower mantle (50 percent) than that found in this study (8 percent). It was 365 suggested by Ohta et al. (2012), that the difference between iron-free and iron-bearing 366 phases observed in lower pressure measurements (<26 GPa) by Manthilake et al. (2011) 367 should be diminished at lower mantle pressures where iron will exist in a low-spin state. 368 Enrichment in aluminum is expected to have a smaller effect, because of the similarity in 369 atomic mass of aluminum to magnesium and silicon. This is assumption is supported by 370 the results of Ohta et al. (2014), but conflicts with those of Manthilake et al. (2011). 
371 Lateral homogeneity in thermal conductivity at the core-mantle boundary is significant, 372 because it implies a direct relationship between the temperature in the lower-most mantle, 373 which can be inferred from seismology (Kustowski et al., 2008), and lateral variations in 374 the heat-flux across the core-mantle boundary, via Fourier's law (Nakagawa and Tackley, 375 2008). Combining Fourier's law for the heat-flux $q=k \partial T / \partial z$, with a linear temperature 376 profile near the core-mantle boundary $T=T_{C M B}-h \partial T / \partial z$, and scaling of shear-wave velocity 377 to temperature $\xi=\left(\partial \mathrm{n} V_{S} / \partial T\right)_{z}$ we find lateral variations in the heat-flux $d q=-k / h \operatorname{dn} V s / \xi$, 378 where $T$ is temperature, $z$ is depth, $T_{C M B}$ is the temperature at the core-mantle boundary, $h$ 379 is height above the core-mantle boundary, and $V_{S}$ is shear-wave veloctiy. To compute $d q$ 380 we use our value of $k$ for pyrolite at the core-mantle boundary, $d \ln V_{S}$ from seismic 381 tomography at $2800 \mathrm{~km}$ depth, giving $h=90 \mathrm{~km}$, and the scaling $\xi$ from a thermodynamic 382 model (Stixrude and Lithgow-Bertelloni, 2011). We do not include the influence of the 383 perovskite to post-perovskite transition on $\xi$ because the pressure at which the transition 384 occurs is uncertain. We find substantial lateral variation in heat-flux, comparable in 385 magnitude to the mean value (Fig. 5). The heat-flux varies from zero (i.e. no heat escaping 386 the core) to nearly twice the mean value. Such large later variations in heat-flux have 387 important implications for our understanding of the origin of Earth's magnetic field (Olson 388 and Christensen, 2002), its behaviour during reversals (Glatzmaier et al., 1999), and the 389 structure of the inner core (Gubbins et al., 2011). Geodynamo simulations (Gubbins et al., 390 2011) indicate that even for much smaller lateral variation in heat flow than we estimate, 391 localized melting of the inner core may occur, which may help to explain anomalies in the 392 structure of the inner core including its radial structure, anisotropy, and hemispherical 393 dichotomy. We note that our estimates of the lateral variation of heat flux are based on the 394 assumption that lateral variations in seismic wave velocity are purely thermal in origin. An 
395 understanding of the contributions of lateral variations in chemistry to the tomographic 396 signal will be important for understanding the pattern of heat flow.

\section{6. Conclusions}

398 In conclusion, our calculations suggest that the lattice thermal conductivity of $\mathrm{MgSiO}_{3}$

399 perovskite depends strongly on pressure, but that temperature and compositional

400 dependence is weak in the deep mantle. Combining our results with seismic tomography,

401 we find large lateral variations in the heat-flux from the core that have important

402 implications for core dynamics. Our predictions of the thermal conductivity provide a firm

403 basis from which further to explore the influence of mantle chemical heterogeneity on the 404 coupled thermal evolution of core and mantle. 


\section{Acknowledgements}

408 This research was supported by the European Research Council under Advanced Grant 409 No. 291432 "MoltenEarth" (FP7/2007-2013), NERC grant number NE/K006290/1 and the 410 National Science Foundation through TeraGrid resources provided by the Texas 411 Advanced Computing Center, under NSF grant EAR080017. In addition, we also 412 acknowledge the use of high performance computing provided by Advanced Research 413 Computing at the University of Leeds and HECToR, the UK's national high-performance 414 computing service, which is provided by UoE HPCx Ltd at the University of Edinburgh, 415 Cray Inc and NAG Ltd, and funded by the Office of Science and Technology through 416 EPSRC's High End Computing Programme. The authors thank Carolina Lithgow-Bertelloni 417 for assistance preparing Fig. 5, and the editor and two anonymous reviewers for 418 constructive comments. S.S. thanks Michael Ammann, John Brodholt, David Dobson, and 419 Andrew Walker for useful discussions. 


\section{References}

Ammann, M.W., Walker, A.M., Stackhouse, S., Wookey, J., Forte, A.M., Brodholt, J.P., Dobson, D.P., 2014. Variation of thermal conductivity and heat flux at the Earth's core mantle boundary. Earth Planet. Sci. Lett. 390, 175-185.

Boghosian, H.H., Dubey, K.S., 1978. Role of Electron-Phonon Interaction and Peripheral Phonons in the Lattice Thermal Conductivity of Doped Semiconductor at Low Temperatures. Phys. Stat. Sol. 88, 417-427.

Chen, Y., Chernatynskiy, A., Brown, D., Schelling, P.K., Artacho, E., Phillpot, S.R., 2012. Critical assessment of classical potentials for $\mathrm{MgSiO} 3$ perovskite with application to thermal conductivity. Phys. Earth Planet. Inter. 210-211, 75-89.

Davies, G.F., 2007. Mantle regulation of core cooling: A geodynamo without core radioactivity? Phys. Earth Planet. Inter. 160, 215-229.

de Koker, N., 2010. Thermal conductivity of MgO periclase at high pressure: Implications for the D" region. Earth Planet. Sci. Lett. 292, 392-398.

Dekura, H., Tsuchiya, T., Tsuchiya, J., 2013. Ab initio Lattice Thermal Conductivity of MgSiO3 Perovskite as Found in Earth's Lower Mantle. Phys. Rev. Lett. 110, 025904.

Dubuffet, F., Yuen, D.A., Rabinowicz, M., 1999. Effects of a realistic mantle thermal conductivity on the patterns of 3-D convection. Earth Planet. Sci. Lett. 171, 401409.

Dziewonski, A.M., Lekic, V., Romanowicz, B.A., 2010. Mantle Anchor Structure: An argument for bottom up tectonics. Earth Planet. Sci. Lett. 299, 69-79.

Fischer, R.A., Campbell, A.J., Lord, O.T., Shofner, G.A., Dera, P., Prakapenka, V.B., 2011. Phase transition and metallization of $\mathrm{FeO}$ at high pressures and temperatures. Geophys. Res. Lett. 38, L24301. 
Flyvbjerg, H., Petersen, H., 1989. Error-estimates on averages of correlated data. J. Chem. Phys. 91, 461-466.

Glatzmaier, G.A., Coe, R.S., Hongre, L., Roberts, P.H., 1999. The role of the Earth's mantle in controlling the frequency of geomagnetic reversals. Nature 401, 885-890.

Goncharov, A.F., Haugen, B.D., Struzhkin, V.V., Beck, P., Jacobsen, S.D., 2008. Radiative conductivity in the Earth's lower mantle. Nature 456, 231-234.

Goncharov, A.F., Struzhkin, V.V., Montoya, J.A., Kharlamova, S., Kundargi, R., Siebert, J., Badro, J., Antonangeli, D., Ryerson, F.J., Mao, W., 2010. Effect of composition, structure, and spin state on the thermal conductivity of the Earth's lower mantle. Phys. Earth Planet. Inter. 180, 148-153.

Gubbins, D., Sreenivasan, B., Mound, J., Rost, S., 2011. Melting of the Earth's inner core. Nature 473, 361-U53.

Haigis, V., Salanne, M., Jahn, S., 2012. Thermal conductivity of MgO, MgSiO3 perovskite and post-perovskite in the Earth's deep mantle. Earth Planet. Sci. Lett. 355-356, $102-108$.

Hofmeister, A.M., 2008. Inference of high thermal transport in the lower mantle from laserflash experiments and the damped harmonic oscillator model. Phys. Earth Planet. Inter. 170, 201-206.

Hofmeister, A.M., 2010. Thermal diffusivity of oxide perovskite compounds at elevated temperature. J. Appl. Phys. 107, 103532.

Howell, P.C., 2011a. Thermal Conductivity Calculation with the Molecular Dynamics Direct Method II: Improving the Computational Efficiency. J. Comput. Theor. Nanosci. 8, 2144-2154.

Howell, P.C., 2011b. Thermal Conductivity Calculation with the Molecular Dynamics Direct Method I: More Robust Simulations of Solid Materials. J. Comput. Theor. Nanosci. 8, 2129-2143. 
Howell, P.C., 2012. Comparison of molecular dynamics methods and interatomic potentials for calculating the thermal conductivity of silicon. J. Chem. Phys. 137, 224111.

Hu, L., Evans, W.J., Keblinski, P., 2011. One-dimensional phonon effects in direct molecular dynamics method for thermal conductivity determination. J. Appl. Phys. $110,113511$.

Imada, S., Ohta, K., Yagi, T., Hirose, K., Yoshida, H., Nagahara, H., 2014. Measurements of lattice thermal conductivity of $\mathrm{MgO}$ to core-mantle boundary pressures. Geophys. Rev. Lett. 41, 060423.

Keppler, H., Dubrovinsky, L.S., Narygina, O., Kantor, I., 2008. Optical Absorption and Radiative Thermal Conductivity of Silicate Perovskite to 125 Gigapascals. Science 322, 1529-1532.

Klemens, P., 1960. Thermal resistance due to point defects at high temperatures. Phys. Rev. 119, 507-509.

Kresse, G., Furthmuller, J., 1996a. Efficiency of ab-initio total energy calculations for metals and semiconductors using a plane-wave basis set. Comput. Mater. Sci. 6 , $15-50$.

Kresse, G., Furthmuller, J., 1996b. Efficient iterative schemes for ab initio total-energy calculations using a plane-wave basis set. Phys. Rev. B 54, 11169-11186.

Kustowski, B., Ekstrom, G., Dziewonski, A.M., 2008. Anisotropic shear-wave velocity structure of the Earth's mantle: A global model. J. Geophys. Res.-Solid Earth 113, B06306.

Lay, T., Hernlund, J., Buffett, B.A., 2008. Core-mantle boundary heat flow. Nat. Geosci. 1, 25-32.

Liao, B., Qiu, B., Zhou, J., Huberman, S., Esfarjani, K., Chen, G., 2015. Significant Reduction of Lattice Thermal Conductivity by the Electron-Phonon Interaction in 

$114,115901$.

Manga, M., Jeanloz, R., 1996. Implications of a metal-bearing chemical boundary layer in D” for mantle dynamics. Geophys. Res. Lett. 23, 3091-3094.

Manthilake, G.M., de Koker, N., Frost, D.J., McCammon, C.A., 2011. Lattice thermal conductivity of lower mantle minerals and heat flux from Earth's core. Proc. Natl. Acad. Sci. U. S. A. 108, 17901-17904.

Marquardt, H., Ganschow, S., Schilling, F.R., 2009a. Thermal diffusivity of natural and

Marquardt, H., Speziale, S., Reichmann, H.J., Frost, D.J., Schilling, F.R. 2009b. Singlecrystal elasticity of $\left(\mathrm{Mg}_{0.9}, \mathrm{Fe}_{0.1}\right) \mathrm{O}$ to $81 \mathrm{GPa}$. Earth. Planet. Sci. Lett. 3-4, 345-352.

Muller-Plathe, F., 1997. A simple nonequilibrium molecular dynamics method for calculating the thermal conductivity. J. Chem. Phys. 106, 6082-6085.

Nakagawa, T., Tackley, P.J., 2008. Lateral variations in CMB heat flux and deep mantle seismic velocity caused by a thermal-chemical-phase boundary layer in 3D spherical convection. Earth Planet. Sci. Lett. 271, 348-358.

Ni, S.D., Tan, E., Gurnis, M., Helmberger, D., 2002. Sharp sides to the African superplume. Science 296, 1850-1852.

Nieto-Draghi, C., Avalos, J.B., 2003. Non-equilibrium momentum exchange algorithm for molecular dynamics simulation of heat flow in multicomponent systems. Mol. Phys. 101, 2303-2307.

Nose, S., 1984. A unified formulation of the constant temperature molecular dynamics methods. J. Chem. Phys. 81, 511-519.

Ohta, K., Yagi, T., Hirose, K., 2014. Thermal diffusivities of MgSiO3 and Al-bearing MgSiO3 perovskites. Am. Miner. 99, 94-97. 
Ohta, K., Yagi, T., Taketoshi, N., Hirose, K., Komabayashi, T., Baba, T., Ohishi, Y., Hernlund, J., 2012. Lattice thermal conductivity of MgSiO3 perovskite and postperovskite at the core-mantle boundary. Earth Planet. Sci. Lett. 349-350, 109-115.

Olson, P., Christensen, U.R., 2002. The time-averaged magnetic field in numerical dynamos with non-uniform boundary heat flow. Geophys. J. Int. 151, 809-823.

Osako, M., Ito, E., 1991. Thermal-diffusivity of MgSiO3 perovskite. Geophys. Res. Lett. 18, 239-242.

Perdew, J., Zunger, A., 1981. Self-interaction correction to density-functional approximations for many-electron systems. Phys. Rev. B 23, 5048-5079.

Rost, S., Garnero, E.J., Williams, Q., Manga, M., 2005. Seismological constraints on a possible plume root at the core-mantle boundary. Nature 435, 666-669.

Roufosse, M., Klemens, P., 1973. Thermal-conductivity of complex dielectric crystals. Phys. Rev. B 7, 5379-5386.

Roufosse, M., Klemens, P., 1974. Lattice thermal-conductivity of minerals at hightempeatures. J. Geophys. Res. 79, 703-705.

Schelling, P.K., Phillpot, S.R., Keblinski, P., 2002. Comparison of atomic-level simulation methods for computing thermal conductivity. Phys. Rev. B 65.

Sellan, D.P., Landry, E.S., Turney, J.E., McGaughey, A.J.H., Amon, C.H., 2010. Size effects in molecular dynamics thermal conductivity predictions. Phys. Rev. B 81.

Stackhouse, S., Stixrude, L., 2010. Theoretical Methods for Calculating the Lattice Thermal Conductivity of Minerals, in: Wentzcovitch, R., Stixrude, L. (Eds.), Theoretical and Computational Methods in Mineral Physics: Geophysical Applications. Mineralogical Soc. Amer., Chantilly, pp. 253-269.

Stackhouse, S., Stixrude, L., Karki, B.B., 2010. Thermal Conductivity of Periclase (MgO) from First Principles. Phys. Rev. Lett. 104. 
Stixrude, L., Lithgow-Bertelloni, C., 2011. Thermodynamics of mantle minerals - II. Phase equilibria. Geophys. J. Int. 184, 1180-1213.

Tadano, T., Gohda, Y., Tsuneyuki, S., 2014. Anharmonic force constants extracted from first-principles molecular dynamics: applications to heat transfer simulations. J. Phys.-Condes. Matter 26, 225402.

Tang, X., Ntam, M.C., Dong, J., Rainey, E.S.G., Kavner, A., 2014. The thermal conductivity of Earth's lower mantle. Geophysical Research Letters 41, 2746-2752.

Tsuchiya, T., Wentzcovitch, R.M., da Silva, C.R.S., de Gironcoli, S., 2006. Spin transition in magnesiowustite in earth's lower mantle. Phys. Rev. Lett. 96.

Wentzcovitch, R.M., Justo, J.F., Wu, Z., da Silva, C.R.S., Yuen, D.A., Kohlstedt, D., 2009. Anomalous compressibility of ferropericlase throughout the iron spin cross-over. Proc. Natl. Acad. Sci. U. S. A. 106, 8447-8452.

Wookey, J., Stackhouse, S., Kendall, J.M., Brodholt, J., Price, G.D., 2005. Efficacy of the post-perovskite phase as an explanation for lowermost-mantle seismic properties. Nature 438, 1004-1007.

Zhou, X.W., Aubry, S., Jones, R.E., Greenstein, A., Schelling, P.K., 2009. Towards more accurate molecular dynamics calculation of thermal conductivity: Case study of 
a

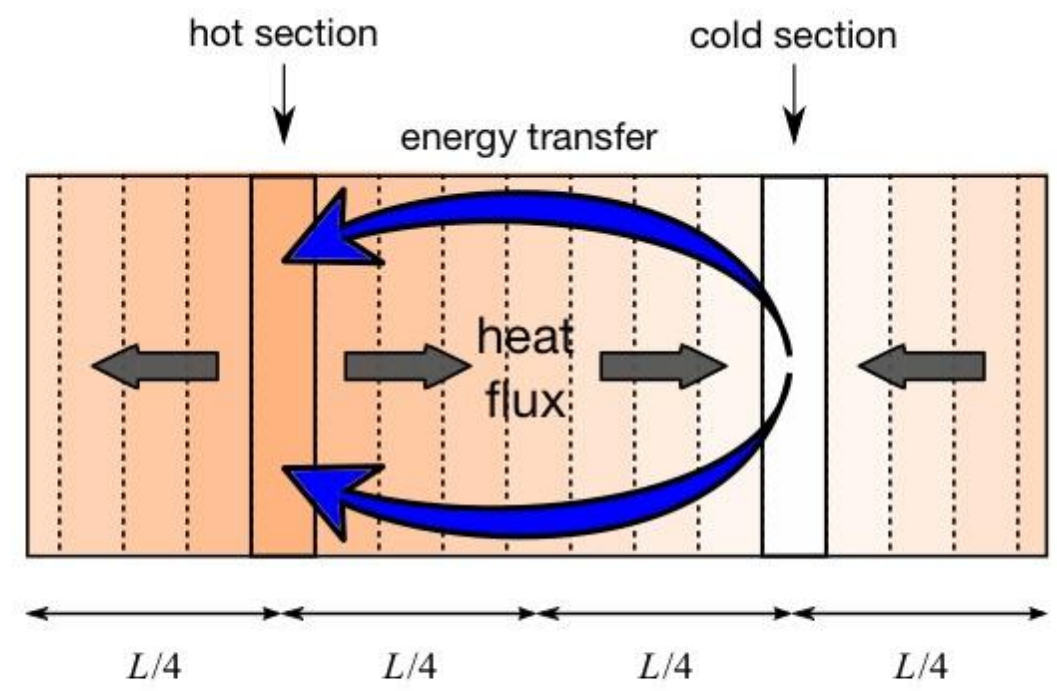

b

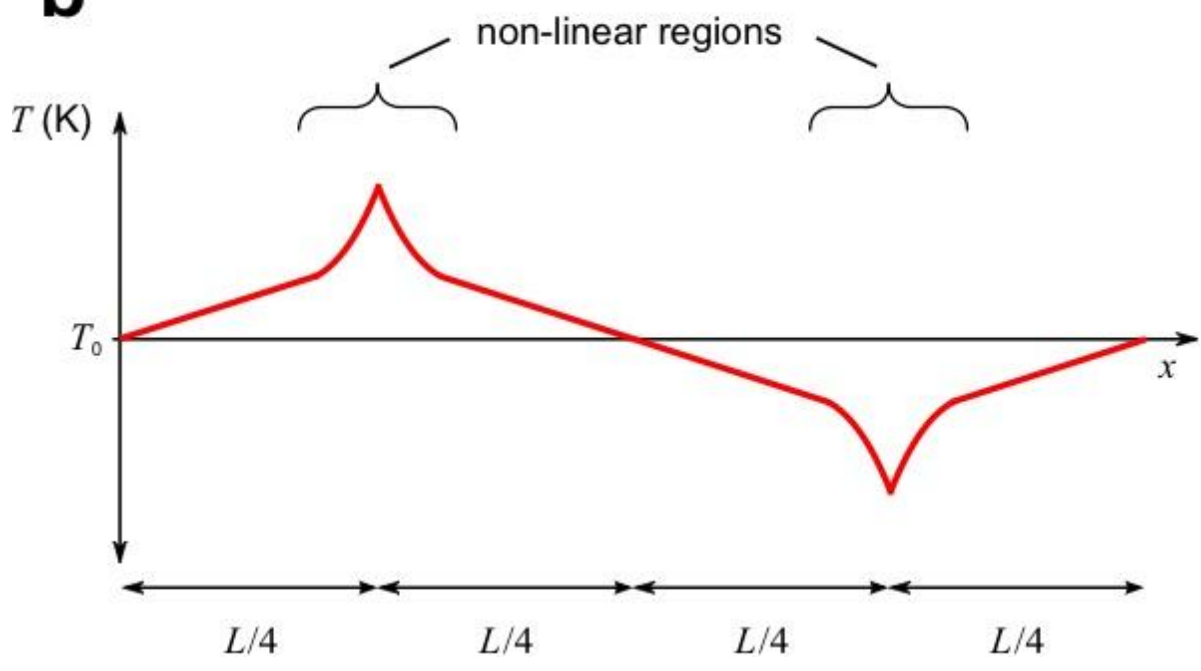

Fig. 1. The non-equilibrium molecular dynamics method for calculating lattice thermal conductivity. The simulation cell is split into sections of equal width. At regular intervals energy is transferred from the 'cold section' to the 'hot section', by means of a virtual elastic collision between the hottest atom in the cold section and coldest atom in the hot section. Due to the periodic nature of the simulation, two temperature gradients form. The non-Newtonian nature of the virtual elastic collision means that the temperature gradient is non-linear around the hot and cold sections. Figure after Stackhouse and Stixrude (2010). 


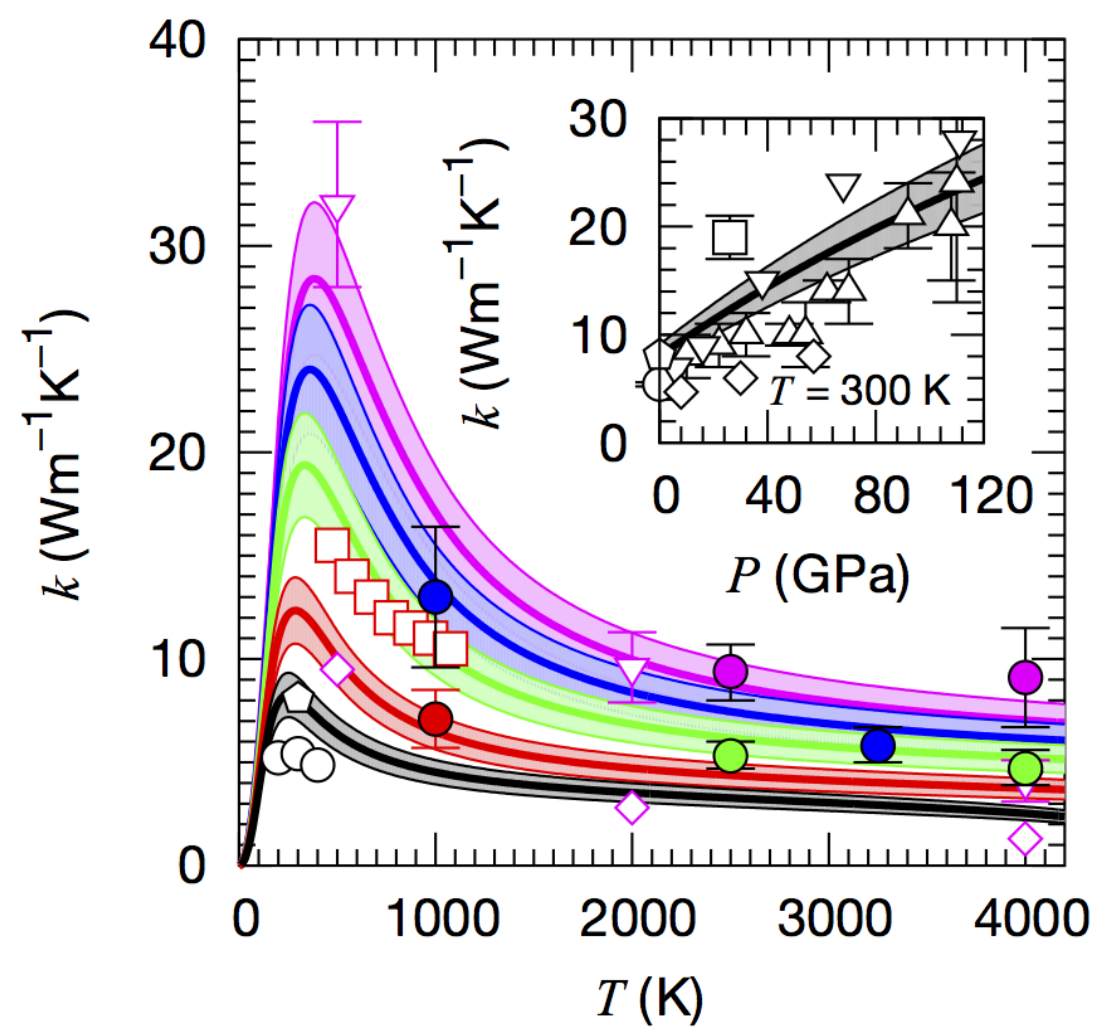

587 Fig. 2. Lattice thermal conductivity of $\mathrm{MgSiO}_{3}$ perovskite as a function of temperature at 588 various pressures (main) and a function of pressure at $300 \mathrm{~K}$ (inset). Filled circles are the 589 results of our NEMD simulations and lines are the values predicted by our model 590 (described in the main text), with shading and error bars indicating standard error. Empty 591 symbols are measured ( $\bigcirc$ Osako and Ito (1991), $\square$ Manthilake et al. (2011), $\triangle$ Ohta et al. 592 (2012), $\square$ Ohta et al. (2014)) and calculated ( $\nabla$ Dekura et al. (2013), $\diamond$ Tang et al. (2014)) 593 values from previous studies. Color code for the main figure: black: $0 \mathrm{GPa}$, red:26 GPa, 594 green:75 GPa, blue:110 GPa and pink:145 GPa. 


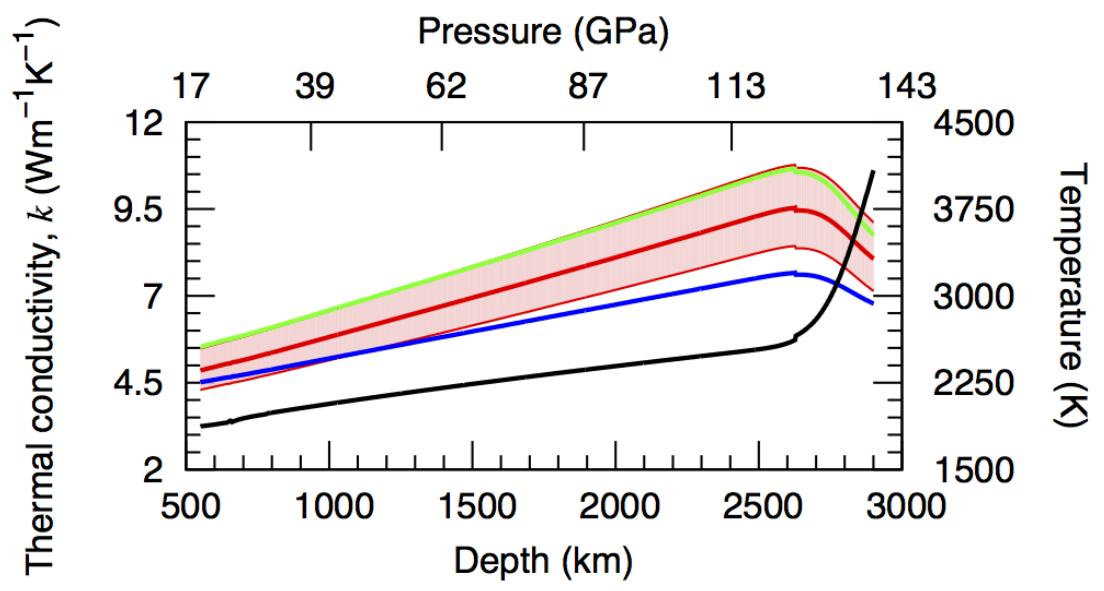

598 Fig. 3. Lattice thermal conductivity of a pyrolite mantle as a function of depth (red line, with 599 the shading indicating standard error). Our results for pure $\mathrm{MgSiO}_{3}$ perovskite (blue line) 600 and iron-free pyrolite (green line) are shown for comparison. The geotherm (black line) is 601 taken from a thermodynamic model (Stixrude and Lithgow-Bertelloni, 2011). 


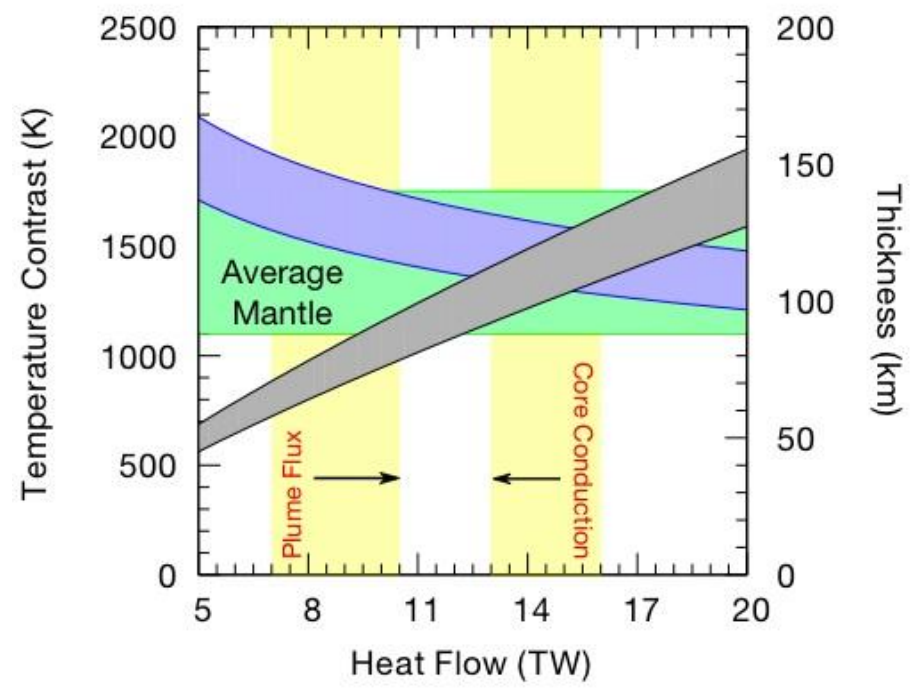

604 Fig. 4. Boundary layer analysis of heat flow. Temperature contrast $\Delta T$ (grey band) and

605 thickness $\delta$ (blue band) of the thermal boundary layer plotted against core-mantle

606 boundary heat flow, with the shading indicating the standard error in our determination of

607 the thermal conductivity (Supplementary Material Section S4). The green shaded area

608 denotes constraints on the temperature contrast across the core-mantle boundary. The

609 orange regions highlight constraints on the heat flow at the core-mantle boundary: upper

610 bound derived from the heat conducted down the core adiabat and lower bound derived

611 from the plume heat-flux and the factor 2-3 increase in the plume thermal anomaly with

612 depth (Davies, 2007). 


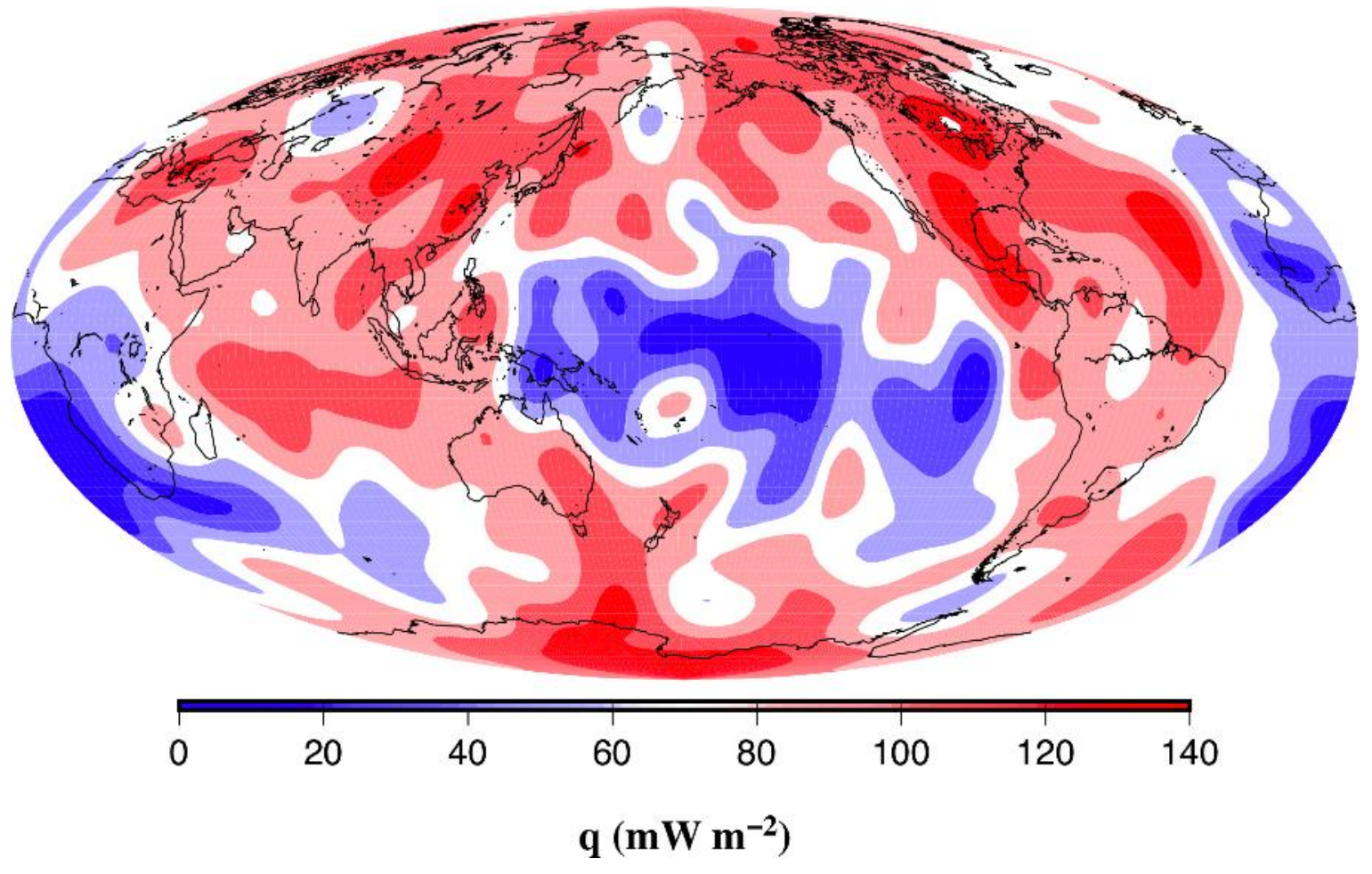

614

616 Fig. 5. Heat-flow at the core-mantle boundary. Values calculated from our estimate of

617 thermal conductivity and the seismic tomographic model of Kustowski et al. (2008) at 2800 $618 \mathrm{~km}$ depth.

619 